\title{
Neuroradiology training in EU: international survey of 31 countries within UEMS frame
}

\author{
Francesca B. Pizzini ${ }^{* *}$ (D) Marek Sasiadek ${ }^{2}$, Francesco Tanzi ${ }^{3}$ and Paolo Ricci ${ }^{4}$
}

\begin{abstract}
Objective: To assess the current framework of interventional and diagnostic neuroradiology in Europe

Methods: The UEMS (European Union of Medical Specialists) Section of Radiology and the subspecialty UEMS Division of Neuroradiology collected by e-mail a survey on the situation of diagnostic and Interventional Neuroradiology' training and practice in Europe. The questionnaire was sent to the national delegates from 31 UEMS member countries, belonging to the European Union, the European Economic Area and the Council of Europe. In case of uncertain or discordant replies, the survey envisaged the involvement of neuroradiology scientific societies' experts for providing a decisive answer.
\end{abstract}

Results: A formal post-residency training in diagnostic and interventional neuroradiology is provided respectively by 12/31 and 20/31 of the European countries. Currently, for becoming neuroradiologist in a country without fellowship program, a radiologist should (1) get subspecialty credits, (2) follow training inside national or international neuroradiology departments, or (3) perform the main reporting activity in neuroradiology. In nearly $2 / 3$ of the States included in the survey, the neurointerventional procedures are provided by radiologists $(22 / 31)$ and in the most frequent scenario a specific training in neurovascular is required to all radiologist or nonradiologist candidates (18/31).

Conclusions: The European framework of neuroradiology's training and practice that emerged through this survey is fragmented, but there is an increasing attention by European scientific societies and institutions to create a common path of training and practice that can guarantee high educational and patient care standards.

Keywords: Neuroradiology, Training, Survey

\section{Key points}

- Neuroradiological (diagnostic and interventional) training in Europe is extremely heterogeneous.

- A survey of European situation is the base for a common action.

- A European neuroradiological training strategy can guarantee higher medical practice and assistance.

\footnotetext{
* Correspondence: francesca.pizzini@aovr.veneto.it

'UEMS Division of Neuroradiology, Radiology, Dept. of Diagnostic and Public Health, Verona University, Piazzale L.A. Scuro 10, 37134 Verona, Italy

Full list of author information is available at the end of the article
}

\begin{abstract}
Introduction
The European Union (EU) training system in diagnostic and interventional neuroradiology is heterogeneous and reflects the different national educational and training structures. European Union of Medical Specialists (UEMS), European Society of Neuroradiology-Diagnostic and Interventional (ESNR), and European Society of Minimally Invasive Neurological Therapy (ESMINT) have recently created a "European Charter for Interventional Neuroradiology" [1, 2], and during this last decade, scientific societies have updated the neuroradiology training skill, knowledge, competences, and attitudes within the European Training curriculum [3] and have been increasing the coordination of training courses and are implementing standardized exams in neuroradiology [4].
\end{abstract}

Springer Open (c) The Author(s). 2020 Open Access This article is licensed under a Creative Commons Attribution 4.0 International License, which permits use, sharing, adaptation, distribution and reproduction in any medium or format, as long as you give appropriate credit to the original author(s) and the source, provide a link to the Creative Commons licence, and indicate if changes were made. The images or other third party material in this article are included in the article's Creative Commons licence, unless indicated otherwise in a credit line to the material. If material is not included in the article's Creative Commons licence and your intended use is not permitted by statutory regulation or exceeds the permitted use, you will need to obtain permission directly from the copyright holder. To view a copy of this licence, visit http://creativecommons.org/licenses/by/4.0/. 
Despite this positive trend, in EU, there is still no official supervising institution, as the Accreditation Council for Graduate Medical Education (ACGME) in the United States of America (USA), which sets common standards of training and periodically reviews them. While in Europe the path of an aspiring neuroradiologist is not standardized, in the USA, after the completion of an ACGME-accredited radiology residency program, a candidate can start an additional training for subspecialty expertise [5]. Fellowships are offered in the USA in both diagnostic and interventional neuroradiology; the first one is ACMGE regulated, and it is a prerequisite (for 1 year) for the latter. To date, only few neurointerventional programs in the USA have pursued the ACGME accreditation pathway, for different reasons [5]. But an alternative accreditation and certification program have been proposed under the aegis of the Committee for Advanced Subspecialty Training (CAST) of the Society of Neurological Surgeons (SNS) [5].

Since there is currently increasing attention to guarantee a widespread and high-level training and practice across all countries, we launched this survey under the UEMS umbrella to investigate the European situation, intending to outline and summarize the variegated reality and hoping that this will lead to a synergistic educational and clinical practice response.

\section{Materials and methods}

The survey was realized within the European Union of Medical Specialists (UEMS) Radiology Section and Neuroradiology Division, and it was focused on the situation of diagnostic and interventional neuroradiology training and practice in Europe, in view of the approval of the European Training Requirements in Interventional Neuroradiology (April 13, 2019) [1, 2].

It was launched in September 2018, and it targeted the national delegates from 31 UEMS member countries-from 1 to 3 responders for each country-and in case of multiple and discordant replies, it was also sent to scientific societies referents provided by European or national neuroradiology societies for a final decision (i.e., France, Italy, Switzerland and UK, asterisked in Table 1). The questionnaire highlighted how significant differences in neuroradiology and interventional training-this latter in endovascular procedures as treatment of aneurysms, acute ischemic stroke, extracranial and intracranial angioplasty/stenting, and embolization of arteriovenous malformation and fistulas and of external carotid (as listed in [1, 2]) - and practice still persist in Europe. As for general categorization of responses, the format (Fig. 1) was a two-choice (yes or no for questions number $1,4,5$, and 6 ) or a multiple- choice (for questions number 2 and 3) questionnaire which covered and synthetized the main possible scenarios.

Regarding the 2 open questions (2. "How to become Neuroradiologist?" and 3. "How to become Interventional Neuroradiologist?"), these following three different scenarios have been envisaged and proposed:

\section{No specific Neuroradiology (NR)/Interventional} Neuroradiology (INR) requirements or qualification: this answer indicates that there is not a NR/INR fellowship or specialty or program, but the participation to NR/INR courses is encouraged for being considered NR or INR.

2. Specific NR/INR requirements, no qualification, when subspecialty credits or training inside neuroradiology departments (national or international) or a main neuroradiology reporting activity are required, but there is not a specific fellowship or specialistic degrees required.

3. Specific NR/INR requirements and qualification, when a recognized fellowship or specialistic degree and training program are required.

The survey has been circulating by e-mail among respondents for 11 months after the launch, and it has been further presented and discussed in the UEMS Section and Division meetings held in March (Vienna) and September (Rome) 2019, then completed and finalized after this latter one.

\section{Results}

The total number of the UEMS countries involved was 31 , and each country provided a unique answer. The survey was sent to 53 UEMS delegates, but, in case of uncertain answer or of discordant answers, it was also forwarded to the scientific societies' experts. The results of the inquiry are summarized in Table 1 and in the main conceptual maps (Figs. 2, 3, and 4), and they are also visible on the UEMS neuroradiology division website: https://neuro.uemsradiology.eu/specialty-in-europe/ \#education-and-training

\section{Neuroradiology training (questions 1 and 2)}

A neuroradiology specialty school, a fellowship, or a specific training program is required in 12 of 31 countries (Fig. 2).

In 10 nations, a school or fellowship is provided, while in the UK and Ireland there is a specific neuroradiology training to follow. Greece will be probably one of the next countries to get a specific NR training, since it is in the process of approving the subspecialty of diagnostic and interventional neuroradiology. 
Table 1 Survey results

\begin{tabular}{|c|c|c|c|c|c|c|c|}
\hline Country & $\begin{array}{l}\text { No. of } \\
\text { respondents }\end{array}$ & $\begin{array}{l}\text { Specialty } \\
\text { school/ } \\
\text { fellowship in } \\
\text { neuroradiology }\end{array}$ & $\begin{array}{l}\text { How to become } \\
\text { neuroradiologist }\end{array}$ & $\begin{array}{l}\text { How to become } \\
\text { interventional } \\
\text { neuroradiologist }\end{array}$ & $\begin{array}{l}\text { Neuroendovascular } \\
\text { procedures } \\
\text { performed by } \\
\text { interventional } \\
\text { radiologists }\end{array}$ & $\begin{array}{l}\text { Neuroendovascular } \\
\text { procedures } \\
\text { performed by non- } \\
\text { radiologists }\end{array}$ & $\begin{array}{l}\text { INR training } \\
\text { required }\end{array}$ \\
\hline Austria & 3 & No & $\begin{array}{l}\text { No specific NR } \\
\text { requirements or } \\
\text { qualification. }\end{array}$ & $\begin{array}{l}\text { No specific INR } \\
\text { requirements or } \\
\text { qualification. } \\
\text { Certification of } \\
\text { neuro- } \\
\text { interventionalists } \\
\text { and training sites } \\
\text { based on existing } \\
\text { modular certifica- } \\
\text { tion concept by the } \\
\text { scientific societies } \\
\text { ÖGIR (Austrian soci- } \\
\text { ety for interven- } \\
\text { tional radiology) } \\
\text { and ÖGNR (Austrian } \\
\text { society for neurora- } \\
\text { diology) is highly } \\
\text { recommended. }\end{array}$ & Yes & Yes & No \\
\hline Belgium & 2 & No & $\begin{array}{l}\text { No specific NR } \\
\text { requirements or } \\
\text { qualification. }\end{array}$ & $\begin{array}{l}\text { No specific INR } \\
\text { requirements or } \\
\text { qualification. }\end{array}$ & Yes & Yes & No \\
\hline Bulgaria & 2 & No & $\begin{array}{l}\text { No specific NR } \\
\text { requirements or } \\
\text { qualification. }\end{array}$ & $\begin{array}{l}\text { Specific INR } \\
\text { requirements, but } \\
\text { No qualification. }\end{array}$ & Yes & Yes & $\begin{array}{l}\text { No, but it is in } \\
\text { progress. }\end{array}$ \\
\hline Croatia & 1 & Yes & $\begin{array}{l}\text { Specific NR } \\
\text { requirements \& } \\
\text { qualification. }\end{array}$ & $\begin{array}{l}\text { No specific INR } \\
\text { requirements or } \\
\text { qualification. }\end{array}$ & Yes & No & $\begin{array}{l}\text { Yes, but no } \\
\text { formal }\end{array}$ \\
\hline Cyprus & 1 & Yes & $\begin{array}{l}\text { Specific NR } \\
\text { requirements, } \\
\text { but No } \\
\text { qualification. }\end{array}$ & $\begin{array}{l}\text { Specific INR } \\
\text { requirements, but } \\
\text { No qualification. }\end{array}$ & Yes & No & Yes \\
\hline $\begin{array}{l}\text { Czech } \\
\text { Republic }\end{array}$ & 1 & $\begin{array}{l}\text { No, it used to } \\
\text { be in the past. }\end{array}$ & $\begin{array}{l}\text { No specific NR } \\
\text { requirements or } \\
\text { qualification. }\end{array}$ & $\begin{array}{l}\text { No specific INR } \\
\text { requirements or } \\
\text { qualification. INR is } \\
\text { part of } \\
\text { Interventional } \\
\text { Radiology training. }\end{array}$ & Yes & No & No \\
\hline Denmark & 1 & No & $\begin{array}{l}\text { No specific NR } \\
\text { requirements or } \\
\text { qualification. }\end{array}$ & $\begin{array}{l}\text { Specific INR } \\
\text { requirements, but } \\
\text { No qualification. }\end{array}$ & Yes & No & $\begin{array}{l}\text { Yes: No formal } \\
\text { fellowship is } \\
\text { available, but } \\
\text { training is } \\
\text { required before } \\
\text { independent } \\
\text { work as INR } \\
\text { specialist is } \\
\text { performed. }\end{array}$ \\
\hline Estonia & 1 & No & $\begin{array}{l}\text { No specific NR } \\
\text { requirements or } \\
\text { qualification. }\end{array}$ & $\begin{array}{l}\text { Specific INR } \\
\text { requirements, but } \\
\text { No qualification. }\end{array}$ & Yes & Yes & Yes \\
\hline Finland & 3 & Yes & $\begin{array}{l}\text { Specific NR } \\
\text { requirements \& } \\
\text { qualification. }\end{array}$ & $\begin{array}{l}\text { Specific INR } \\
\text { requirements, but } \\
\text { No qualification. }\end{array}$ & Yes & No & Yes \\
\hline France* & 3 & No & $\begin{array}{l}\text { Specific NR } \\
\text { requirements, } \\
\text { but No } \\
\text { qualification. }\end{array}$ & $\begin{array}{l}\text { Specific INR } \\
\text { requirements, but } \\
\text { No qualification. }\end{array}$ & Yes & Yes & Yes \\
\hline Germany & 3 & Yes & $\begin{array}{l}\text { Specific NR } \\
\text { requirements \& } \\
\text { qualification. }\end{array}$ & $\begin{array}{l}\text { Specific INR } \\
\text { requirements or } \\
\text { qualification. }\end{array}$ & Yes & No & Yes \\
\hline
\end{tabular}


Table 1 Survey results (Continued)

\begin{tabular}{|c|c|c|c|c|c|c|c|}
\hline Country & $\begin{array}{l}\text { No. of } \\
\text { respondents }\end{array}$ & $\begin{array}{l}\text { Specialty } \\
\text { school/ } \\
\text { fellowship in } \\
\text { neuroradiology }\end{array}$ & $\begin{array}{l}\text { How to become } \\
\text { neuroradiologist }\end{array}$ & $\begin{array}{l}\text { How to become } \\
\text { interventional } \\
\text { neuroradiologist }\end{array}$ & $\begin{array}{l}\text { Neuroendovascular } \\
\text { procedures } \\
\text { performed by } \\
\text { interventional } \\
\text { radiologists }\end{array}$ & $\begin{array}{l}\text { Neuroendovascular } \\
\text { procedures } \\
\text { performed by non- } \\
\text { radiologists }\end{array}$ & $\begin{array}{l}\text { INR training } \\
\text { required }\end{array}$ \\
\hline Greece & 2 & $\begin{array}{l}\text { No. But in the } \\
\text { process of the } \\
\text { subspecialty of } \\
\text { diagnostic and } \\
\text { interventional } \\
\text { neuroradiology } \\
\text { to be approved. }\end{array}$ & $\begin{array}{l}\text { No specific NR } \\
\text { requirements or } \\
\text { qualification. }\end{array}$ & $\begin{array}{l}\text { Specific INR } \\
\text { requirements \& } \\
\text { qualification. The } \\
\text { qualification is } \\
\text { currently obtained } \\
\text { by training in other } \\
\text { countries that } \\
\text { provide INR } \\
\text { qualifications and is } \\
\text { approved by the } \\
\text { Ministry of Health. }\end{array}$ & $\begin{array}{l}\text { No. There is a } \\
\text { discussion that } \\
\text { Interventional } \\
\text { Radiologists get } \\
\text { involved in stroke } \\
\text { after a minimum of } \\
\text { 9-month training. }\end{array}$ & Yes & Yes \\
\hline Hungary & 1 & Yes & $\begin{array}{l}\text { Specific NR } \\
\text { requirements \& } \\
\text { qualification. It is } \\
\text { a secondary } \\
\text { board } \\
\text { examination } \\
\text { following board } \\
\text { exam in } \\
\text { Radiology. }\end{array}$ & $\begin{array}{l}\text { Specific INR } \\
\text { requirements \& } \\
\text { qualification. See } \\
\text { point } 6 .\end{array}$ & $\begin{array}{l}\text { Yes, after } \\
\text { completion of the } \\
\text { special competency } \\
\text { license training and } \\
\text { examination in } \\
\text { Neurointerventions. }\end{array}$ & $\begin{array}{l}\text { Yes, after } \\
\text { completion of the } \\
\text { special competency } \\
\text { license training and } \\
\text { examination in } \\
\text { Neurointerventions. }\end{array}$ & $\begin{array}{l}\text { Yes, it is a special } \\
\text { competency } \\
\text { license, requiring } \\
\text { a } 2 \text { years fulltime } \\
\text { clinical training in } \\
\text { INR following a } \\
\text { board exam in } \\
\text { either radiology, } \\
\text { neuroradiology, } \\
\text { neurology, } \\
\text { neurosurgery or } \\
\text { cardiology. }\end{array}$ \\
\hline Iceland & 1 & No & $\begin{array}{l}\text { Specific NR } \\
\text { requirements \& } \\
\text { qualification. }\end{array}$ & $\begin{array}{l}\text { Specific INR } \\
\text { requirements \& } \\
\text { qualification. }\end{array}$ & No & No & No \\
\hline Ireland & 1 & $\begin{array}{l}\text { Yes: no } \\
\text { specialty school } \\
\text { exists, but } \\
\text { fellowship } \\
\text { training in } \\
\text { Neuroradiology } \\
\text { is provided. }\end{array}$ & $\begin{array}{l}\text { Specific NR } \\
\text { requirements \& } \\
\text { qualification. }\end{array}$ & $\begin{array}{l}\text { Specific INR } \\
\text { requirements, but } \\
\text { No qualification. } \\
\text { Must have } \\
\text { completed } 2 \text { years } \\
\text { of fellowship } \\
\text { training in } \\
\text { neuroradiology. }\end{array}$ & No & No & Yes \\
\hline Italy* & 4 & No & $\begin{array}{l}\text { Specific NR } \\
\text { requirements, } \\
\text { but No } \\
\text { qualification. }\end{array}$ & $\begin{array}{l}\text { Specific INR } \\
\text { requirements, but } \\
\text { No qualification. }\end{array}$ & Yes & Yes & No \\
\hline Latvia & 1 & Yes & $\begin{array}{l}\text { Specific NR } \\
\text { requirements \& } \\
\text { qualification. }\end{array}$ & $\begin{array}{l}\text { Specific INR } \\
\text { requirements, but } \\
\text { No qualification. }\end{array}$ & Yes & No & Yes \\
\hline Lithuania & 1 & No & $\begin{array}{l}\text { No specific NR } \\
\text { requirements or } \\
\text { qualification. }\end{array}$ & $\begin{array}{l}\text { No specific INR } \\
\text { requirements or } \\
\text { qualification. INR } \\
\text { procedures could } \\
\text { be performed by } \\
\text { any interventional } \\
\text { radiologist. }\end{array}$ & Yes & $\begin{array}{l}\text { Yes. In smaller } \\
\text { centres, } \\
\text { cardiologists } \\
\text { perform } \\
\text { thrombectomy } \\
\text { (only) in case of } \\
\text { stroke. }\end{array}$ & No \\
\hline Luxembourg & 1 & No & $\begin{array}{l}\text { Specific NR } \\
\text { requirements, } \\
\text { but No } \\
\text { qualification. }\end{array}$ & $\begin{array}{l}\text { Specific INR } \\
\text { requirements, but } \\
\text { No qualification. }\end{array}$ & Yes & Yes & No \\
\hline Malta & 1 & No & $\begin{array}{l}\text { Specific NR } \\
\text { requirements, } \\
\text { but No } \\
\text { qualification. }\end{array}$ & $\begin{array}{l}\text { Specific INR } \\
\text { requirements, but } \\
\text { No qualification. }\end{array}$ & No & No & Yes \\
\hline Netherlands & 1 & Yes & $\begin{array}{l}\text { Specific NR } \\
\text { requirements \& } \\
\text { qualification. }\end{array}$ & $\begin{array}{l}\text { Specific INR } \\
\text { requirements \& } \\
\text { qualification. }\end{array}$ & $\begin{array}{l}\text { No, except for } \\
\text { endovascular } \\
\text { treatment (stroke). }\end{array}$ & Yes & $\begin{array}{l}\text { Yes (dedicated } \\
\text { fellowship for INR, } \\
\text { which can be }\end{array}$ \\
\hline
\end{tabular}


Table 1 Survey results (Continued)

\begin{tabular}{|c|c|c|c|c|c|c|c|}
\hline Country & $\begin{array}{l}\text { No. of } \\
\text { respondents }\end{array}$ & $\begin{array}{l}\text { Specialty } \\
\text { school/ } \\
\text { fellowship in } \\
\text { neuroradiology }\end{array}$ & $\begin{array}{l}\text { How to become } \\
\text { neuroradiologist }\end{array}$ & $\begin{array}{l}\text { How to become } \\
\text { interventional } \\
\text { neuroradiologist }\end{array}$ & $\begin{array}{l}\text { Neuroendovascular } \\
\text { procedures } \\
\text { performed by } \\
\text { interventional } \\
\text { radiologists }\end{array}$ & $\begin{array}{l}\text { Neuroendovascular } \\
\text { procedures } \\
\text { performed by non- } \\
\text { radiologists }\end{array}$ & $\begin{array}{l}\text { INR training } \\
\text { required }\end{array}$ \\
\hline & & & & & & & $\begin{array}{l}\text { entered by } \\
\text { certified } \\
\text { Radiologists, } \\
\text { Neurologists, and } \\
\text { Neurosurgeons). }\end{array}$ \\
\hline Norway & 1 & No & $\begin{array}{l}\text { No specific NR } \\
\text { requirements or } \\
\text { qualification. }\end{array}$ & $\begin{array}{l}\text { No specific INR } \\
\text { requirements or } \\
\text { qualification. }\end{array}$ & Yes & Yes & No \\
\hline Poland & 3 & No & $\begin{array}{l}\text { No specific NR } \\
\text { requirements or } \\
\text { qualification. }\end{array}$ & $\begin{array}{l}\text { Specific INR } \\
\text { requirements \& } \\
\text { qualification } \\
\text { (certificate provided } \\
\text { by Section of } \\
\text { Interventional } \\
\text { Neuroradiology, } \\
\text { which is a } \\
\text { Subdivision of } \\
\text { Polish Medical } \\
\text { Society of } \\
\text { Radiology). }\end{array}$ & Yes, only stroke. & Yes & Yes \\
\hline Portugal & 1 & Yes & $\begin{array}{l}\text { Specific NR } \\
\text { requirements \& } \\
\text { qualification. } \\
\text { Neuroradiology } \\
\text { is an } \\
\text { independent } \\
\text { speciality with a } \\
\text { five-year training } \\
\text { programme and } \\
\text { a final examin- } \\
\text { ation by a na- } \\
\text { tional jury. }\end{array}$ & $\begin{array}{l}\text { Specific INR } \\
\text { requirements \& } \\
\text { qualification. }\end{array}$ & $\begin{array}{l}\text { No. Only } \\
\text { Neuroradiologists } \\
\text { can perform neuro } \\
\text { intervention. }\end{array}$ & $\begin{array}{l}\text { No. Only } \\
\text { Neuroradiologists } \\
\text { can perform these } \\
\text { interventions as } \\
\text { answered } \\
\text { previously. }\end{array}$ & Yes \\
\hline Romania & 1 & No & $\begin{array}{l}\text { Specific NR } \\
\text { requirements, } \\
\text { but No } \\
\text { qualification. } \\
\text { From } 2018 \text { NR is } \\
\text { part of } \\
\text { specialization of } \\
\text { Interventional } \\
\text { Radiology. }\end{array}$ & $\begin{array}{l}\text { Specific INR } \\
\text { requirements \& } \\
\text { qualification. } \\
\text { Mandatory training } \\
\text { in NR and INR, and } \\
\text { candidates must } \\
\text { pass an exam in } \\
\text { order to perform } \\
\text { Neurointerventional } \\
\text { procedures. }\end{array}$ & Yes & $\begin{array}{l}\text { Yes, very few cases } \\
\text { (neurosurgeons). }\end{array}$ & $\begin{array}{l}\text { Yes, but probably } \\
\text { no more in the } \\
\text { future. }\end{array}$ \\
\hline Slovenia & 1 & No & $\begin{array}{l}\text { Specific NR } \\
\text { requirements, } \\
\text { but No } \\
\text { qualification. }\end{array}$ & $\begin{array}{l}\text { Specific INR } \\
\text { requirements, but } \\
\text { No qualification. }\end{array}$ & Yes & No & Yes \\
\hline Spain & 2 & No & $\begin{array}{l}\text { No specific NR } \\
\text { requirements or } \\
\text { qualification. }\end{array}$ & $\begin{array}{l}\text { Specific INR } \\
\text { requirements, but } \\
\text { No qualification. }\end{array}$ & Yes & Yes & $\begin{array}{l}\text { No. However, } \\
\text { most Neuro- } \\
\text { interventionalists } \\
\text { have received } \\
\text { training from } \\
\text { Interventio-nal } \\
\text { Neuroradiologists } \\
\text { in Academic/Uni- } \\
\text { versity Hospitals. }\end{array}$ \\
\hline Sweden & 2 & Yes & $\begin{array}{l}\text { Specific NR } \\
\text { requirements \& } \\
\text { qualification. }\end{array}$ & $\begin{array}{l}\text { Specific INR } \\
\text { requirements, but } \\
\text { No qualification. }\end{array}$ & No & Yes & Yes \\
\hline Switzerland* & 3 & Yes & $\begin{array}{l}\text { Specific NR } \\
\text { requirements \& }\end{array}$ & $\begin{array}{l}\text { Specific INR } \\
\text { requirements \& }\end{array}$ & $\begin{array}{l}\text { No (unless in a } \\
\text { certified }\end{array}$ & $\begin{array}{l}\text { No (unless in a } \\
\text { certified }\end{array}$ & Yes \\
\hline
\end{tabular}


Table 1 Survey results (Continued)

\begin{tabular}{|c|c|c|c|c|c|c|c|}
\hline Country & $\begin{array}{l}\text { No. of } \\
\text { respondents }\end{array}$ & $\begin{array}{l}\text { Specialty } \\
\text { school/ } \\
\text { fellowship in } \\
\text { neuroradiology }\end{array}$ & $\begin{array}{l}\text { How to become } \\
\text { neuroradiologist }\end{array}$ & $\begin{array}{l}\text { How to become } \\
\text { interventional } \\
\text { neuroradiologist }\end{array}$ & $\begin{array}{l}\text { Neuroendovascular } \\
\text { procedures } \\
\text { performed by } \\
\text { interventional } \\
\text { radiologists }\end{array}$ & $\begin{array}{l}\text { Neuroendovascular } \\
\text { procedures } \\
\text { performed by non- } \\
\text { radiologists }\end{array}$ & $\begin{array}{l}\text { INR training } \\
\text { required }\end{array}$ \\
\hline & & & $\begin{array}{l}\text { qualification \& } \\
\text { examination. }\end{array}$ & $\begin{array}{l}\text { qualification \& } \\
\text { examination. }\end{array}$ & $\begin{array}{l}\text { neurovascular } \\
\text { centre under the } \\
\text { supervision of a } \\
\text { certified } \\
\text { neuroradiologist). }\end{array}$ & $\begin{array}{l}\text { neurovascular } \\
\text { centre under the } \\
\text { supervision of a } \\
\text { certified } \\
\text { neuroradiologist). }\end{array}$ & \\
\hline Turkey & 1 & No & $\begin{array}{l}\text { Specific NR } \\
\text { requirements \& } \\
\text { qualification. }\end{array}$ & $\begin{array}{l}\text { Specific INR } \\
\text { requirements \& } \\
\text { qualification. }\end{array}$ & Yes & Yes & No \\
\hline $\mathrm{UK}^{*}$ & 4 & $\begin{array}{l}\text { Yes: no } \\
\text { specialty school } \\
\text { exists, but } \\
\text { fellowship } \\
\text { training in } \\
\text { Neuroradiology } \\
\text { is provided. }\end{array}$ & $\begin{array}{l}\text { Specific NR } \\
\text { requirements, } \\
\text { but No } \\
\text { qualification. }\end{array}$ & $\begin{array}{l}\text { Specific INR } \\
\text { requirements, but } \\
\text { No qualification. }\end{array}$ & $\begin{array}{l}\text { Not yet. (currently } \\
\text { there are body } \\
\text { interventional } \\
\text { radiologists training } \\
\text { for thrombectomy). }\end{array}$ & No & Yes \\
\hline
\end{tabular}

*Countries where the involvement of additional referents from European or National scientific societies was necessary due to the uncertainty or discordance of the UEMS delegate's answer/s

In countries where there is not a fellowship/school or training (Table 1), the NR expertise could be reached through:

1. Residency subspeciality credits or training inside national or international recognized neuroradiology training centers or if the main work is in neuroradiology (specific NR requirements, no qualification answer)

2. Participation to neuroradiology courses (no specific $N R$ requirements or qualification answer)

Survey "Neuroradiology in Europe"

\section{Is there a Specialty school/Fellowship in Neuroradiology? \\ $\square$ Yes $\square$ No}

\section{How to become Neuroradiologist (NR)?}

a. No specific NR requirements or qualification (e.g. NR courses encouraged)

b. Specific NR requirements, but No qualification (e.g. residency subspeciality credits; training inside Neurorad. DPTs/Abroad; main work in NR) c. Specific NR requirements \& qualification (e.g. NR fellowship; NR speciality)

\section{How to become Interventional Neuroradiologist (INR)?}

a. No specific INR requirements or qualification (e.g. main work in IR; INR courses encouraged)

b. Specific INR requirements, but No qualification (e.g. residency subspeciality credits; Interventional Training inside Neurorad. DPTS/Abroad; main work in INR)

c. Specific INR requirements \& qualification (e.g. INR fellowship/Subspeciality)

\section{Neuroendovascular procedures performed by Interventional Radiologists? \\ $\square$ Yes}

$\square \quad$ No

\section{Neuroendovascular procedures performed by non-Radiologists?}

$\square$ Yes $\square$ No

\section{INR Training required?}

$\square$ Yes $\square$ No

Fig. 1 Template of the survey 


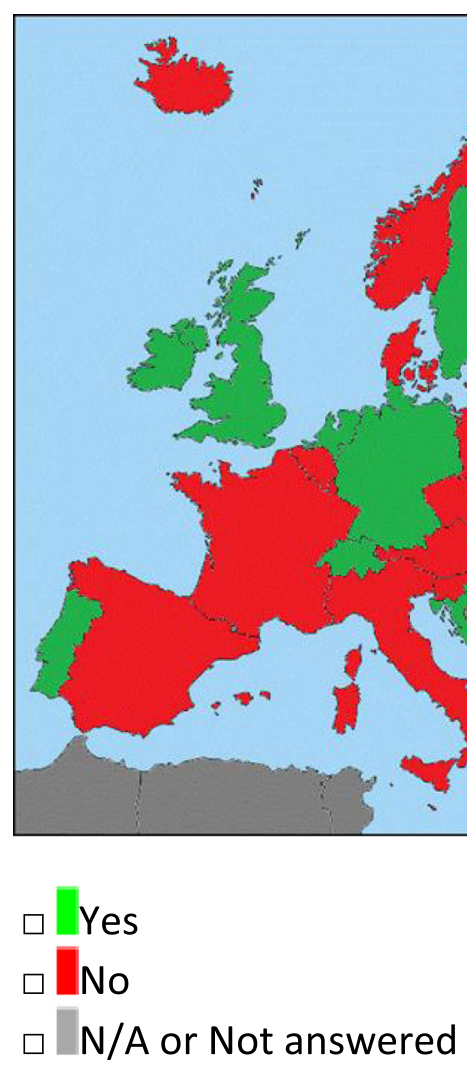

Fig. 2 Question 1: Specialty school/fellowship/training in neuroradiology. In red, the countries where a specialty training does not exist, while in green the ones where it is available. In gray, the countries which have not answered or which were not included in the survey from the beginning

\section{Interventional neuroradiology training and practice (questions 3-6)}

The most frequent scenario (Fig. 3) is the one in which the Interventional Neuroradiology procedures are covered by interventional radiologists (22), with the specificity of Portugal-where only neuroradiologists can perform neurointervention-and of some nations-where there are further requirements (e.g., a specific training in neurointerventions or under NR supervision, as in Hungary and Switzerland) or where the procedures are limited to stroke treatment (i.e., Poland). In 17/31 countries (often only in few centers), neurovascular stroke procedures are performed also by non-radiologist-mainly neurosurgeons or cardiologists (Table 1).

Twenty nations require a specific INR training (Fig. 4; Table 1) before independent work as INR specialist could be performed (i.e., Denmark). This could be also a qualification such as a dedicated fellowship for interventional neuroradiology, which can be entered by certified radiologists, neurologists, and neurosurgeons (The Netherlands), or a special competency license (Hungary).

In all the other countries where the qualification is not required, the INR training could be achieved in a similar way to the NR (i.e., (1) through subspeciality credits or training inside national or international recognized INR
Training centers or if the main workload is in INR-specific INR requirements, no qualification answer-or (2) participation to INR courses-No specific NR requirements or qualification answer-).

\section{Discussion}

This is the first survey to explore the European interventional and diagnostic training in different countries, and the questionnaire development and structure tried to synthesize and cover the main differences-but also to cluster some similarities-between neuroradiology educational programs and practices.

There is a wide variety of training in neuroradiology offering all over the world due to a widely variation of the structure and regulation of the training and, though less, to the variation of its duration [5]. And throughout the world, the main differences of training programs are not limited to neuroradiology, but also to the structure and duration of the medical school curricula and radiology residency programs (teaching modules, duration, a faculty-to-resident ratio) [5].

Regarding the specific training in neuroradiology in Europe, some nations have a specialized fellowship only in interventional or in both diagnostic and interventional (Table 1) with the specificity of Portugal where 

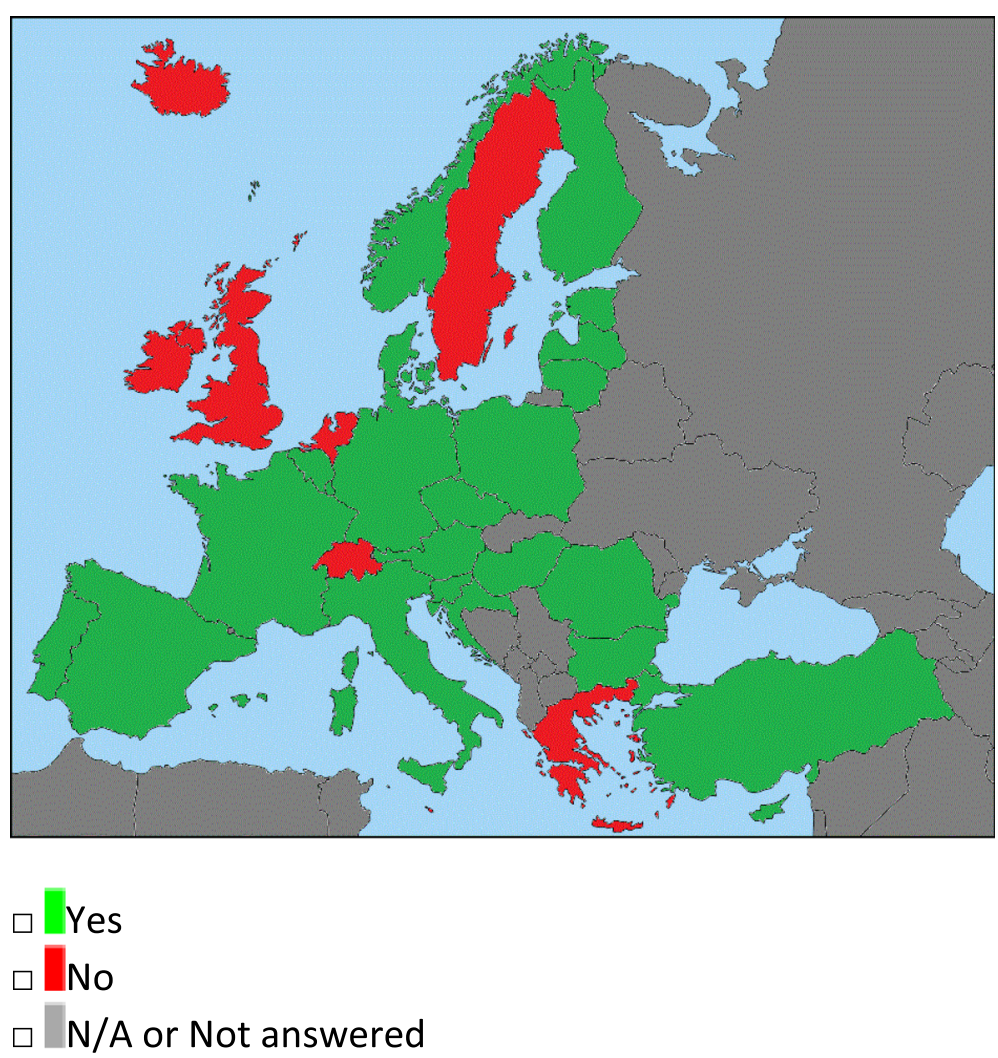

Fig. 3 Question 4: Neuroendovascular procedures performed by interventional radiologists. In green the countries where the interventional procedures are performed by interventional radiologists (in Portugal only by neuroradiologist), while in red, the ones where the procedures are performed by non-radiologist. In gray, the countries which have not answered or which were not included in the survey from the beginning

neuroradiologists follow a separate training track and their residency is separate from the general radiology.

The definition of standards of training and practice can guarantee highest quality medical care and safety. This can be reached by accreditation and certification of training programs. There is a worldwide interest in the production of consensus documents in neurointerventional (NI) training which see the USA as the leader. Different neurosurgical, neuroradiological, and neurologic societies connected to NI have agreed on a training curriculum and related documents [6], proposing individual training, certification and requirements, and resources.

In the USA, there are also structured diagnostic radiology educational programs and neuroradiology fellowship training which are ACGMR-approved and based on concrete guidelines [7-9], including the implementation of national certifying examinations [7].

The survey's results evidenced Europe's lack of guidelines and standardization of training and practice, even if training and educational courses are provided in NI by ESMINT (ECMINT, European Course in Minimally Invasive Neurological Therapy; EXMINT, European Stroke Course; Stroke Winter School and ESMINT fellowship) [10] and ESNR, the latter organizing also diagnostic courses for different levels of learners (i.e., European School of Radiology (ESOR), European Course in Neuroradiology (ECNR), Higher qualifications) [4]. The firstlevel (ESOR) course can be addressed to residents, and it is organized on a yearly basis as a 2-day course, while ECNR is recommended to radiologist with a minimum of neuroradiology training and divided in different modules of learning (1st, embryology/anatomy/malformations/genetics; 2nd, tumors and tumor-like lesions; 3rd, vascular diseases; 4th, trauma/degenerative/metabolic/ inflammatory), while third-level courses are designed for neuroradiologists and provide higher qualification in interventional or spine, pediatric, or head and neck neuroradiology [4].

\section{Generalizability of results}

The limitations of this survey are based on the bias for the responders that were selected within the UEMS and main European societies' representatives. In addition, some answers may not summarize all the complexity of one national reality, and sometimes, there were some differences in the replies from the referents coming from the same country. Anyway, in case of inconsistent answers, we were able to cross-check the validity of these 

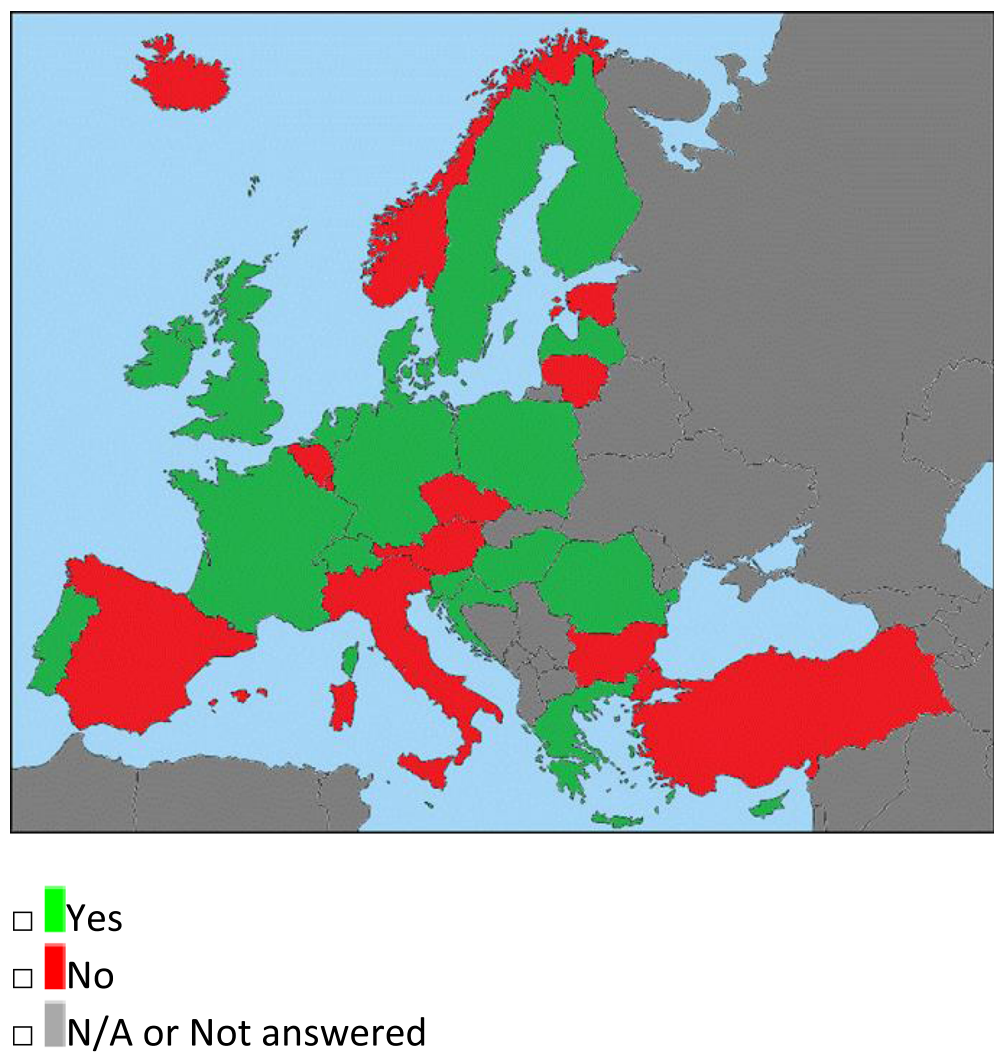

Fig. 4 Question 6: INR training required. In green, the countries where a specific interventional training is required, while in red, the ones where it is not. In gray, the countries which have not answered or which were not included in the survey from the beginning

replies by interviewing national representatives of neuroradiology societies.

In conclusion, this is the first study providing a picture of the current scenario of the neuroradiology training and practice in Europe. Active intervention is needed for harmonizing the heterogeneity of organization of NR training between the EU countries and for creating a common standard of knowledge. That can be achieved through the implementation of a standardized European program and a Charter for educational and training and then through establishment of a common certification and license.

\section{Abbreviations}

ACGME: Accreditation Council for Graduate Medical Education; CAST: Committee for Advanced Subspecialty Training; EDiNR: European Diploma in Neuroradiology; ESMINT: European Society of Minimally Invasive Neurological Therapy; ESNR: European Society of Neuroradiology-Diagnostic and Interventional; ESOR: European School of Radiology; EU: European Union; INR: Interventional neuroradiology; NI: Neurointerventional: NR: Neuroradiology; SNS: Society of Neurological Surgeons; UEMS: Union of Medical Specialists; USA: United States of America

\section{Acknowledgements}

We express our gratitude to the Section of Radiology and the Division of Neuroradiology. Particularly, we would like to thank the following respondents and contributors to the Survey:

Prof. H. Deutschmann-Austria

Dr. J. Trenkler-Austria
Prof. F. Frühwald—Austria

Prof. J. Van Goethem—Belgium

Dr. G. Maleux -Belgium

Prof. N. Traikova-Bulgaria

Prof. D. Zlatareva-Bulgaria

Prof. D. Ozretic-Croatia

Dr. C. Tziakouri-Cyprus

Prof. M. Herman-Czech Republic

Dr. M. Cortsen—Denmark

Dr. M. Reim—Estonia

Prof. R. Vanninen-Finland

Dr. J. Pekkola_Finland

Dr. P. Saari-Finland

Prof. A. Luciani-France

Dr. E. Guillemot-France

Prof. V. Vidal-France

Prof. O. Jansen-Germany

Prof. P. Schramm—Germany

Prof. Vorwerk-Germany

Prof. E. Kapsalaki-Greece

Dr. E. Archontakis—Greece

Prof. I. Szikora-Hungary

Dr. S. Kristjánsson-lceland

Prof. M. Lee-Ireland

Dr. F. Causin-Italy

Dr. M. Cariati-Italy

Dr. M. Muto-Italy

Dr. F. B. Pizzini-Italy

Prof. G. Krumina_Latvia

Prof. S. Lukosevicius_-Lithuania

Dr. R. Demuth—Luxembourg

Dr. R. Grech-Malta

Prof. M. Smits—-The Netherlands 
Dr. G. A. Ringstad—Norway

Prof. M. Sasiadek_Poland

Dr. A. Zimny-Poland

Dr. A. Majos-Poland

Dr. G. Sá Nunes_-Portugal

Prof. B. Popa-Romania

Prof. K. Surlan Popovic_-Slovenia

Dr. A. Cabrera Zubizarreta_-Spain

Dr. M. Serrallonga-Spain

Dr. P. Brouwer-Sweden

Prof. H. Stahlbrandt-Sweden

Prof. S. Qanadli-Switzerland

Prof. G. Schroth—Switzerland

Prof. S. Özbek-Turkey

Dr. J. Kraft-UK

Dr. K. Lobotesis—UK

Dr. R. Lenthall—UK

Dr. W. Ramsden—UK

\section{Statement of data access and integrity}

The authors declare that they had full access to all of the data in this study, and the authors take complete responsibility for the integrity of the data and the accuracy of the data collection.

\section{Authors' contributions}

The conception of the study was done by Prof. Paolo Ricci. The study design was done by all the authors. Data collection and analysis was done by Dr. Francesca Benedetta Pizzini (F.B.P.) and Mr. Francesco Tanzi (F.T.). First drafting was done by FBP and revised by Prof. Marek Sasiadek. Editing and final approval was done by all the authors.

\section{Funding}

None

\section{Availability of data and materials}

The datasets used and/or analyzed during the current study are available online https://neuro.uemsradiology.eu/specialty-in-europe/\#education-andtraining

\section{Ethics approval and consent to participate}

Not applicable

\section{Consent for publication}

Yes

\section{Competing interests}

The authors declare that they have no competing interests.

\section{Author details}

'UEMS Division of Neuroradiology, Radiology, Dept. of Diagnostic and Public Health, Verona University, Piazzale L.A. Scuro 10, 37134 Verona, Italy. ${ }^{2}$ UEMS Division of Neuroradiology, Department of General and Interventional Radiology and Neuroradiology, Wroclaw Medical University, Wroclaw, Poland. ${ }^{3}$ UEMS Section of Radiology, Brussels, Belgium. ${ }^{4}$ UEMS Section of Radiology, Department of Radiology, Sapienza University of Rome, Rome, Italy.

Received: 19 February 2020 Accepted: 4 May 2020

Published online: 22 May 2020

\section{References}

1. Sasiadek M, Kocer N, Szikora I et al (2019) Standards for European training requirements in interventional neuroradiology guidelines by the Division of Neuroradiology/Section of Radiology European Union of Medical Specialists (UEMS), in cooperation with the Division of Interventional Radiology/UEMS, the European Society of Neuroradiology (ESNR), and the European Society of Minimally Invasive Neurological Therapy (ESMINT). J Neurointerv Surg. https://doi.org/10.1136/neurintsurg-2019-015537

2. Sasiadek M, Kocer N, Szikora I et al (2020). Standards for European training requirements in interventional neuroradiology: Guidelines by the Division of Neuroradiology/Section of Radiology European Union of Medical Specialists (UEMS), in cooperation with the Division of Interventional Radiology/UEMS, the European Society of Neuroradiology (ESNR), and the European Society of Minimally Invasive Neurological Therapy (ESMINT). Neuroradiology. https://doi.org/10.1007/s00234-019-02300-2.

3. European Training Curriculum for Radiology- ESR-UEMS supported. https:// www.uems.eu/_data/assets/pdf_file/0018/72270/UEMS-2018.37-EuropeanTraining-Requirements-in-Radiology.pdf. Accessed January 2020

4. European Society of Neuroradiology, diplomas and exams. https://www. esnr.org/en/diplomas-exams/. Accessed January 2020.

5. Schneider T, Huisman TA, Fiehler J, Savic L, Yousem DM (2015) Differences in neuroradiology training programs around the world. AJNR Am J Neuroradiol. https://doi.org/10.3174/ajnr.A4132

6. Day AL, Siddiqui AH, Meyers PM et al (2017) Training standards in neuroendovascular surgery program accreditation and practitioner certification, training standards in neuroendovascular surgery: program accreditation and practitioner certification. Stroke. https://doi.org/10.1161/ STROKEAHA.117.016560

7. ACGME program requirements for graduate medical education in diagnostic radiology.http://www.acgme.org/Portals/O/PFAssets/ ProgramRequirements/420_DiagnosticRadiology_2019_TCC.pdf?ver=201903-28-091211-973. Accessed July 2019.

8. ACGME Diagnostic Radiology Case log categories and required minimum numbers. https://www.acgme.org/Portals/O/PFAssets/ProgramResources/ DR_Case_Log_Categories.pdf? Accessed November 2018.

9. ACGME Interventional Radiology Case Log Categories and Required Minimum Numbers. https:/www.acgme.org/Portals/0/PFAssets/ ProgramResources/IR_Case_Log_Categories.pdf?ver=2018-12-20-135106-953. Accessed December 2018.

10. ESMINT, training and educational. https://www.esminteu/trainingeducation/. Accessed January 2020

\section{Publisher's Note}

Springer Nature remains neutral with regard to jurisdictional claims in published maps and institutional affiliations.

\section{Submit your manuscript to a SpringerOpen ${ }^{\circ}$ journal and benefit from:}

- Convenient online submission

- Rigorous peer review

- Open access: articles freely available online

- High visibility within the field

- Retaining the copyright to your article

Submit your next manuscript at $\boldsymbol{\nabla}$ springeropen.com 